\title{
SELF-STABILIZING FAult TOLERANCE Distributed Cyber Physical Systems
}

\author{
Ammar Alhaj Ali, Said Krayem, Bronislav Chramcov \& Mohamad Fadlallah Kadi
}
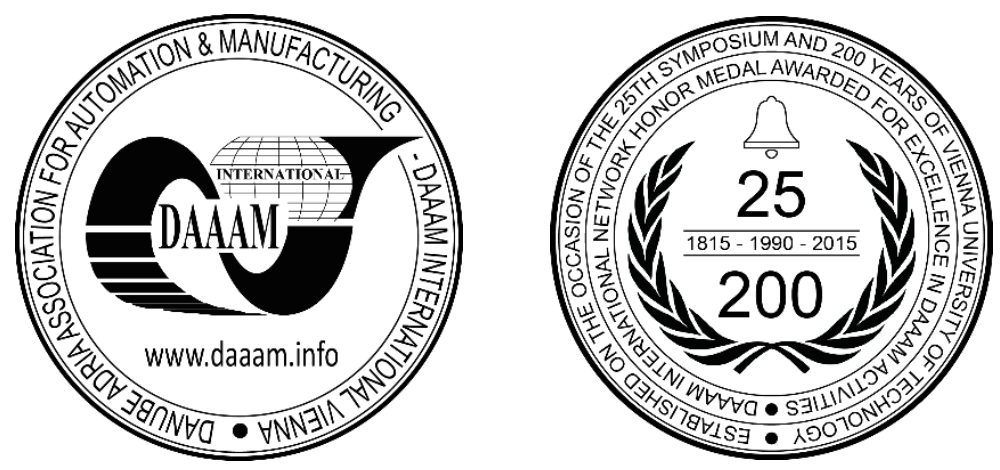

This Publication has to be referred as: Ali, A[lhaj] A[mmar]; Krayem, S[aid]; Chramcov, B[ronislav] \& Kadi, M[ohamad] F[adlallah] (2018). Self-Stabilizing Fault Tolerance Distributed Cyber Physical Systems, Proceedings of the 29th DAAAM International Symposium, pp.1173-1180, B. Katalinic (Ed.), Published by DAAAM International, ISBN 978-3-902734-20-4, ISSN 1726-9679, Vienna, Austria

DOI: $10.2507 / 29$ th.daaam.proceedings.169

\begin{abstract}
Distributed Cyber-physical systems (DCPS) represent a new field in automatic control and recently they are increasingly used in life critical system, where the probability of tragic failure has to be kept below very low levels. In other side, fault tolerance has been used to face failures and achieve best performance of systems. Thus, it becomes critical to develop models that are tolerant toward the failure of system. This will enable guarantee of DCPS that will continue to run even through failure status. In this paper we take self-stabilizing as an example of fault tolerance in distributed cyber physical systems, where self-stabilization provides non-masking approach to fault tolerance. Then the Event-B approach is presented as formal method for system-level modelling and analysis. It is proposed using the Rodin modelling tool for Event-B that integrates modelling and proving.
\end{abstract}

Keywords: fault tolerance; self-stabilizing system; distributed cyber physical system; modelling; Event-B

\section{Introduction}

The development and design of a DCPS requires a huge effort of reasoning with respect to many challenges, and accurate performance and reliability requirements. Hence, dependability and reliability are two important factors for any DCPS design. However, the potential failures in any component of system may prevent DCPS from performing dependable and reliable operations. Such these failures may occur in any part in DCPS (the cyber part, the physical part, sensors or actuators) must be analyzed and take into account at the early stages of design and build the system [1].

The major problem of achieving dependable DCPS is approached using a system engineering process to gain an understanding of the problem domain, since fault tolerance cannot be solved only as a software problem due to the nature of DCPS, which includes close coordination among hardware, software and physical objects [2].

Various fault-tolerant algorithms have been proposed in order to increase system stability. The paper [14] considers a method of synthesis of identification system for faults appearing in the various elements of complex mechatronic systems described by nonlinear differential equations. In the paper [15] a special feedback by the signal of residual is introduced in the diagnostic observers obtained with the logic-dynamic approach to solve the problem of identification of values of faults. 
In this paper, we propose self-stabilizing as an approach of fault tolerance in DCPS. Self-stabilization is a compensating mechanism for systems prone to faults which are either too expensive or impossible to eliminate. When a fault occurs, and is detected, the system automatically takes steps to return itself to a state from which the fault has been removed. The "fault-free" state is considered stable in the sense that an absolute-correctness argument has established [3].

The paper is organized as follows. In section 2 the related works are presented, section 3 gives a brief outline of the cyber physical system and architecture of DCPS, and in section 4 we briefly introduce faults in distributed systems and classification of faults, in section 5, we describe fault tolerance and approaches used in fault tolerance. Then section 6 presents a self-stabilizing as an approach of fault tolerance. Section 7 gives a brief outline of Event B and section 8 offers our case study. Section 9 describes modelling in Event-B of our case study and finally in section 10 our conclusions are presented.

\section{Related works}

In this section we will discuss how self-stabilizing fault tolerance distributed systems has been dealt with in the literature. For more references on related topics; there is an excellent survey on self-stabilization in the distributed systems by Abusayeed Saifullah [4]. This report surveys most previous works available in the literature of self-stabilizing systems, and presents a good number of works pertaining self-stabilization distributed systems, which were proposed in last years. Most of them are very recent.

Taylor T Johnson [5] offers analyzing fault-tolerance in DCPS through the presentation of two case studies. In each case study, the DCPS are modelled as distributed algorithms executed by a set of agents, where each agent acts independently based on information obtained from its communication neighbours and agents may suffer from various failures.

Thesis [6] focuses on the fault tolerance in distributed systems using self-stabilization. This work presents a collection of self-stabilizing algorithms for well-known problems in distributed systems, and presents the first set of self-stabilizing algorithms with safe convergence property for packing and alliance problems in arbitrary network graphs.

\section{Cyber physical systems}

The term cyber-physical system is one of the new buzzwords in the engineering community. It originates from the need to have a denomination for a new category of embedded systems where the emphasis was made on the increased interactions between the physical part and the computational part of the system [7].

Cyber-physical systems represent an interconnection between processing systems and physical systems through communication, sensor and actuator technologies, see figure 1. The application domains are rapidly increasing like medical, automotive, aircraft and even military systems. However, in spite all the advantages introduced by this control approach there is also a key issue - the fault control system [8].

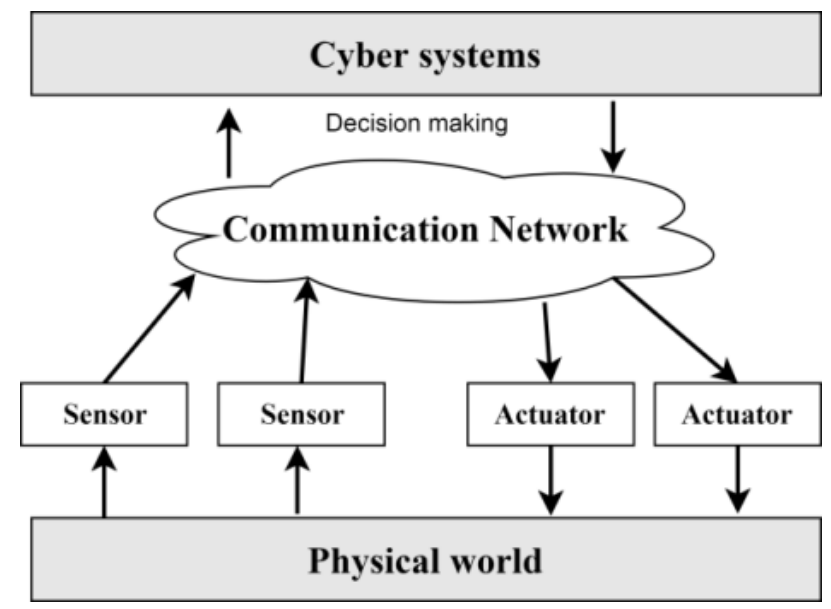

Fig. 1. Cyber physical system view

\section{Faults in distributed systems}

In everyday language, the terms fault, failure, and error are used interchangeably. A fault (or failure) can be either a hardware defect or a software/programming mistake (bug). In contrast, an error is a manifestation of the fault/failure/bug. Computer scientists and engineers have responded to the challenge of designing complex systems with a variety of tools and techniques to reduce the number of faults in the systems they build. However, that is not enough, because we need to build systems that will acknowledge the existence of faults as a fact of life [9]. 
Faults in distributed systems can be classified according to several aspects. Regarding their duration, faults can be classified into:

1. Transient faults: Faults that are arbitrary in nature can strike the system, but there is a point in the execution beyond which these faults no longer occur.

2. Permanent faults: Faults that are arbitrary in nature can strike the system, but there is a point in the execution beyond which these faults always occur.

3. Intermittent faults: Faults that are arbitrary in nature can strike the system at any moment in the execution [10].

Another classification of hardware faults is into benign and malicious faults. A fault that just causes a unit to go dead is called benign, such faults are the easiest to deal with. Far more insidious are the faults that cause a unit to produce reasonable-looking, but incorrect, output, or that make a component "act maliciously" and send differently valued outputs to different receivers [9].

\section{Fault tolerance}

Fault tolerance is the property that enables a system to continue with its correct operation even in the presence of faults (errors), and it is generally implemented by error detection and subsequent system recovery. Fault tolerance has been a subject of research for a long time, and significant amount of work has been produced over the years [11].

The fault tolerance approaches can be classified as masking and non-masking. A masking fault tolerance approach aims at masking the effects of the faults using redundancy (additional hardware or software). Such an approach is able to make the system service always available, but the redundancy may considerably increase the cost of the system. The nonmasking fault tolerance approach is relatively cheap, but the drawback of this approach is that it accepts the temporary unavailability of the system service for a limited time [6].

On other hand, we can classify fault-tolerant algorithms to two major categories:

1. Robust algorithms: These use redundancy on several levels of information, of communications, or of the system's nodes, in order to overlap to the extent that the rest of the code can safely be executed. They usually rely on the hypothesis that a limited number of faults will strike the system, so as to preserve at least a majority of correct elements (sometimes more if the faults are more severe). Typically, these are masking algorithms

2. Self-stabilizing algorithms: These rely on the hypothesis that the faults are transient (in other words, limited in time), but do not set constraints regarding the extent of the faults. An algorithm is self-stabilizing if it manages, in a finite time, to present an appropriate behaviour independent from the initial state of its elements, meaning that the variables of the elements may exist in a state that is arbitrary (and impossible to achieve by running the application normally).Typically, self-stabilizing algorithms are non-masking, because between the moment when the faults cease and the moment when the system has stabilized to an appropriate behaviour, the execution may turn out to be somewhat erratic.

With a self-stabilizing algorithm, an external user may experience erratic behaviour (the stabilizing phase) after the faults have actually ceased, while a robust algorithm will always appear as behaving properly. In contrast, a selfstabilizing algorithm makes no assumption about the extent or the nature of the faults, while robust systems will generally put constraints on those [10], see figure 2.
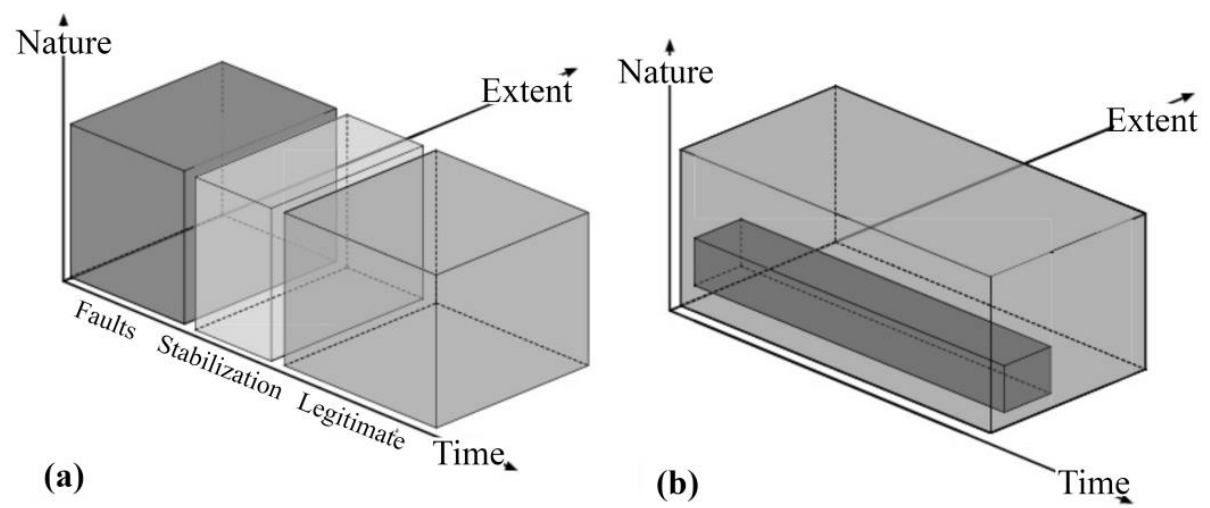

Fig. 2. Self stabilization (a) vs. robustness (b)

\section{Self-stabilizing system}

Self-stabilization, a non-masking fault tolerance approach, is an optimistic paradigm to provide autonomous resilience against an unlimited number of transient faults in the distributed systems [6]. 
A system is self-stabilizing if it can start from any possible configuration and converges to a desired configuration (legitimate configuration) in finite time by itself without using any external intervention. Convergence is also guaranteed when the system is affected by transient faults. This makes self-stabilization an elegant approach for transient faulttolerance. Figure 3 illustrates the behaviour of self-stabilization system. Note that self-stabilizing system may not reach a legitimate configuration (or desired configuration) if faults occur frequently during the convergence. For this reason, most publications assume that all faults are transient, i.e. no further faults occur during the stabilization of the system [12].

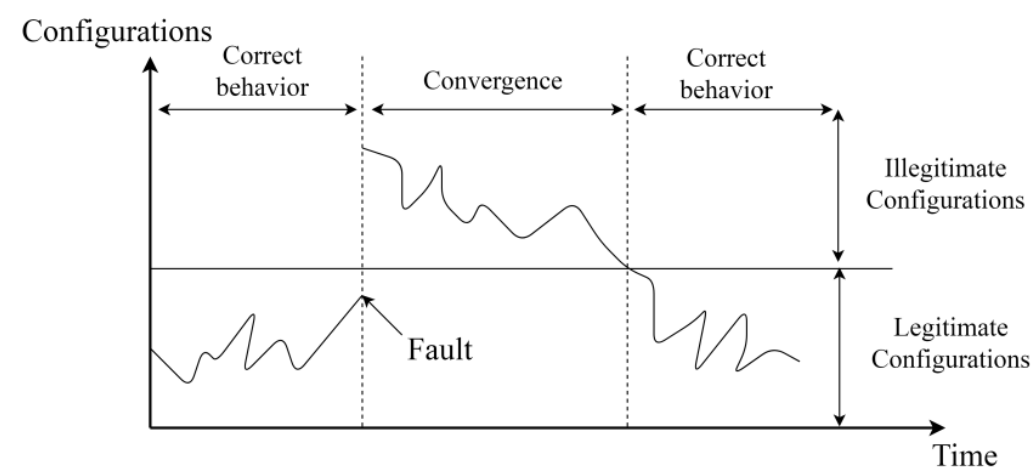

Fig. 3. Self-stabilizing system's behaviour

We define self-stabilization for a system $S$ with respect to a predicate $P$, over its set of global states, where $P$ is intended to identify its correct execution. $S$ is self-stabilizing with respect to predicate $P$ if it satisfies the following two properties:

- Closure: $P$ is closed under the execution of $S$. That is, once $P$ is established in $S$, it cannot be falsified.

- Convergence: Starting from an arbitrary global state, $S$ is guaranteed to reach a global state satisfying $P$ within a finite number of state transitions.

The goal in a self-stabilizing distributed system is to start from an arbitrary (possibly illegitimate) initial state and then to reach a legitimate state after a finite number of moves (steps). Self-stabilizing algorithms are resilient to transient faults that perturb the state of the system arbitrarily. That is, if unexpected perturbations bring the system from a legitimate state to an illegitimate state, then the system must be able to again reach a legitimate state after a finite number of moves without any external intervention [4].

\section{Event B approach}

Event-B is a mathematical approach for developing formal models of systems. An Event-B model is constructed from a collection of modelling elements. These elements include invariants, events, guards and actions. The modelling elements have attributes that can be based on Set Theory and Predicate Logic. Set Theory is used to represent data-types and to manipulate the data. Logic is used to apply conditions to the data.

The development of an Event-B model goes through two stages; abstraction and refinement, the abstract machine specifies the initial requirements of the system. Refinement is carried out in several steps - with each step adding more detail to the system. The Rodin Platform is an Eclipse-based IDE for Event-B that provides effective support for refinement and mathematical proof. The platform is open source, contributes to the Eclipse framework and is further extendable with plugins.

In Event-B we have two kinds of components (context and machine). Figure 4 shows relationships between machine and context [13].

1. Context: describes the static elements of a model.

2. Machine: describes the dynamic behaviour of a model.

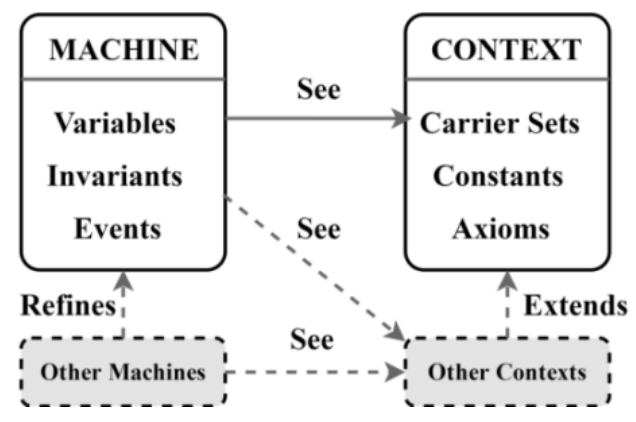

Fig. 4. Machine and Context relationship 


\section{Case study}

Consider a DCPS of N mobile robots; each one has components of a computer with some software processes which representing the interaction of cyber processes of the DCPS, and sensors and actuators representing the interaction with physical processes of the DCPS. These components act on the cyber and physical state. The DCPS is a finite collection of agents ( $\mathrm{N}$ agents), which execute and communicate simultaneously. Each agent exchanges messages with its neighbours. Then, based on these messages, the agents update their software state and decide on a rate-of-change for any continuous variable, see figure 5 .

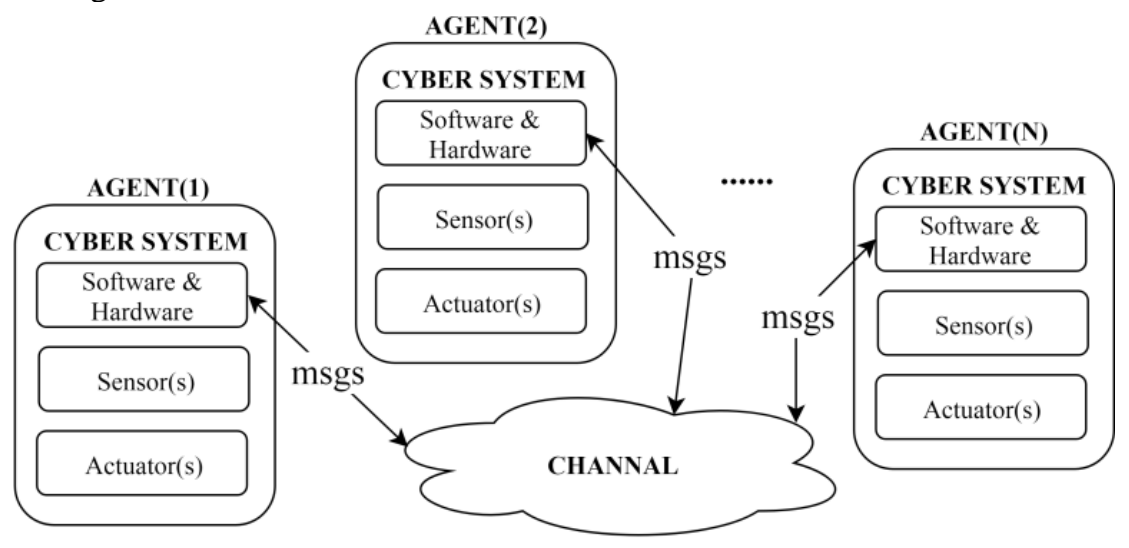

Fig. 5. Typical architecture of a DCPS with $\mathrm{N}$ agents

The DCPS can be represented as a single discrete transition system with a transition that updates some physical and cyber states for all the agents. At the beginning of each round, each agent broadcasts messages. Next each agent receives the messages sent by its neighbours, and finally it computes its local variables based on its local state and the messages collected from its neighbours [5].

\section{Modelling of Self-Stabilizing DCPS as Discrete Transition System in event B}

\subsection{Abstract model}

In our model; we have $N$ mobile robots (agent), each agent has ID. Each agent have set of states and at each round, each agent will update its state according to received messages from its communication neighbours. So we modelled agents with their states and value of each state at each round (execution fragment), and for each agent there is special boolean variable FAIL_AGENT to distinguish between failure-free execution fragment and failure execution, see Source Code 1.

$\mathrm{k} \in \mathbb{N} / / / \mathrm{k}$ rounds
stID $\subseteq 0 \cdot$ Max_StatusID // set of Status
AgentID $\subseteq 0 \cdot \operatorname{Max}$ _AgentID // set of Agents
Status_IDEStID $\rightarrow$ AG_STATES // Value of Status
AGENTS_Status_IDEAgentID $\rightarrow$ Status_ID // the set of states for agent i
AGENTS_Status_ID_REAGENTS_Status_ID $\rightarrow\{\mathrm{k}\} / /$ An execution fragment is sequence of states
FAILED_AGENTEAgentID $\rightarrow$ BOOL

Code 1. Variables and invariants of our model

Failed transition may occur between update transitions due to some exogenous event of specific agent in system ag $\in$ AgentID. In Source Code 2; we can see an execution fragment of system (round) by an alternating sequence of states by abstract event Exec.

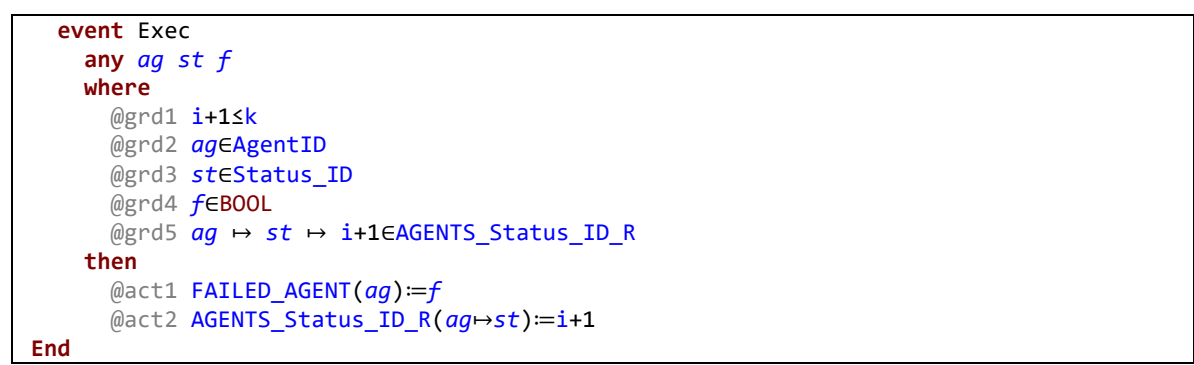

Code 2. A specification of Exec event 


\subsection{Refinement Model}

Let's suppose $S$ is a stable set of states for system, called the legal states. Then system is self-stabilizing for $S$ if and only if there exists a set of states $T$ for system, called the illegal states, such that

- $S \subseteq T$.

- $T$ is invariant.

- $\quad S$ is stable for any failure-free execution, that is, for any non-faulty transition.

- $\quad$ There exists a reachable state in $S$ along any failure-free execution fragment which begins with any state in $T$.

Figure 6 illustrates fault tolerance, where $T$ is an invariant set and $S$ is a stable set, $Q O$ is the set of start states, $E f$ are execution fragments with failed transitions and Eff are failure-free executions.

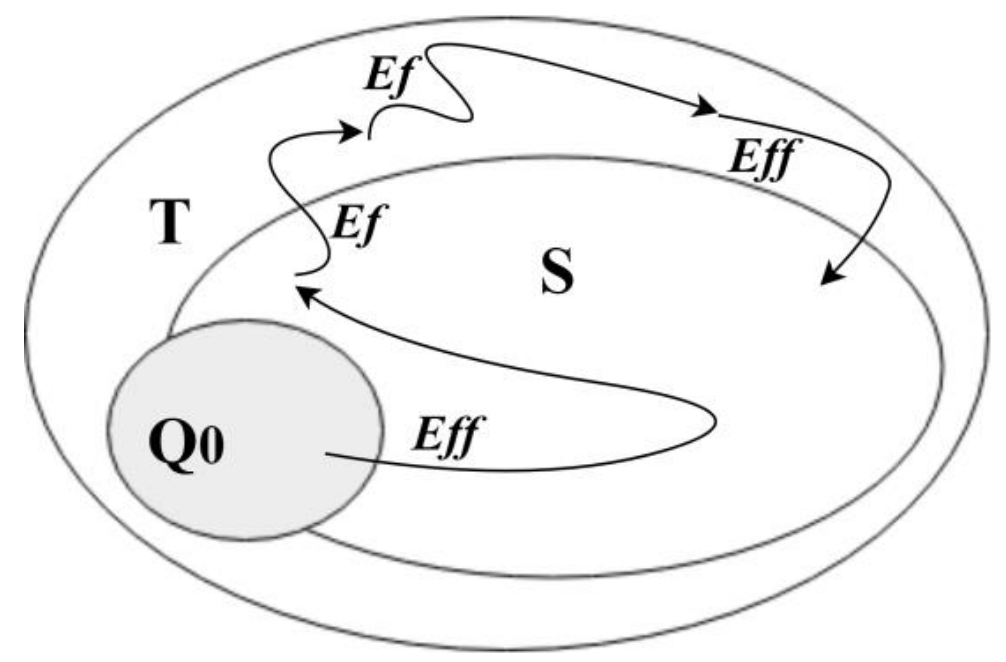

Fig. 6. Fault tolerance by self-stabilizing

In refinement model we will achieve fault tolerance by self-stabilizing algorithm according to the figure 6 . In this case new variable is added; see source code 3 .

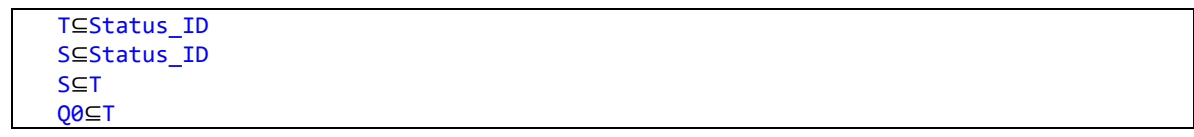

Code 3. New variables and invariants of refinement model.

Then, it is necessary to refine Exec events by four new events:

1. Event is an execution fragment which begins with a start state $s t \in Q O$ and move to state in $S$ and transition is nonfaulty, here we can say the system is stable (see source code 4).

2. Event is an execution fragment which begins with a start state $s t \in S$ and move to state in $T$ and transition is faulty (see source code 5).

3. Event is an execution fragment which begins with a start state $s t \in T$ and move to state in $T$ and transition is faulty (see source code 6).

4. Event is an execution fragment which begins with a start state $s t \in T$ and move to state in $S$ and transition is nonfaulty, here we can say the system is self-stabilizing (see source code 7).

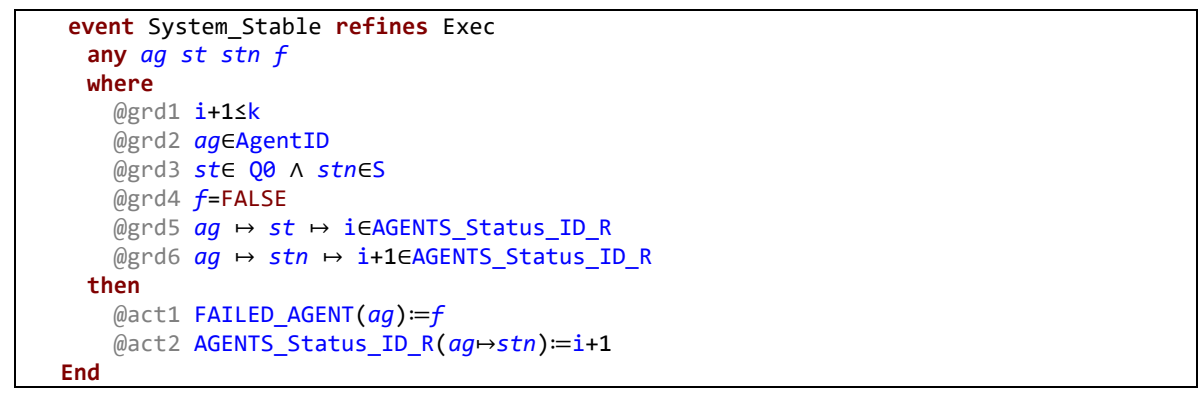

Code 4. A specification of System_Stable event 


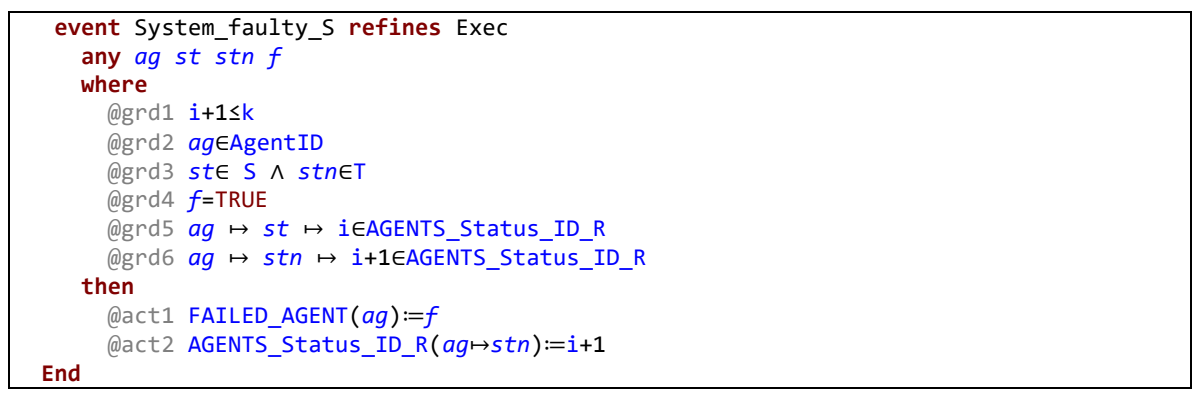

Code 5. A specification of System_faulty_S event

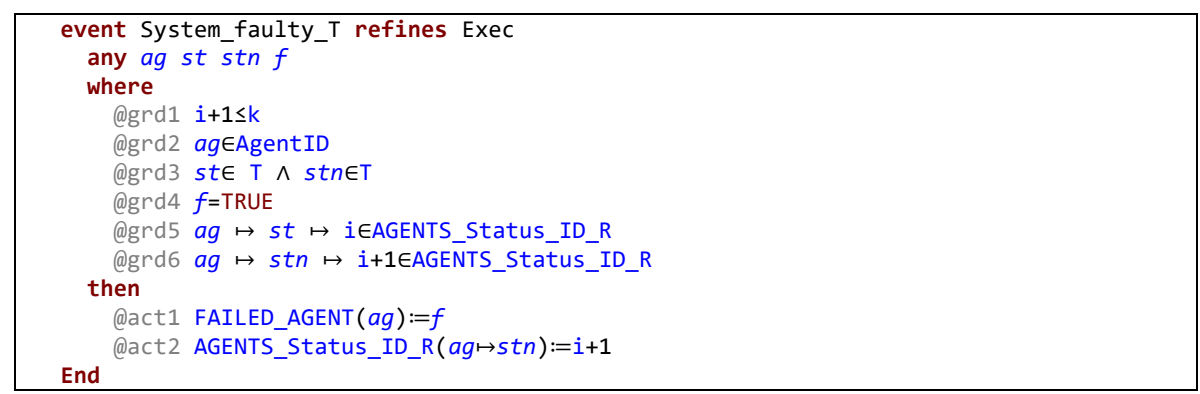

Code 6. A specification of System_faulty_T event

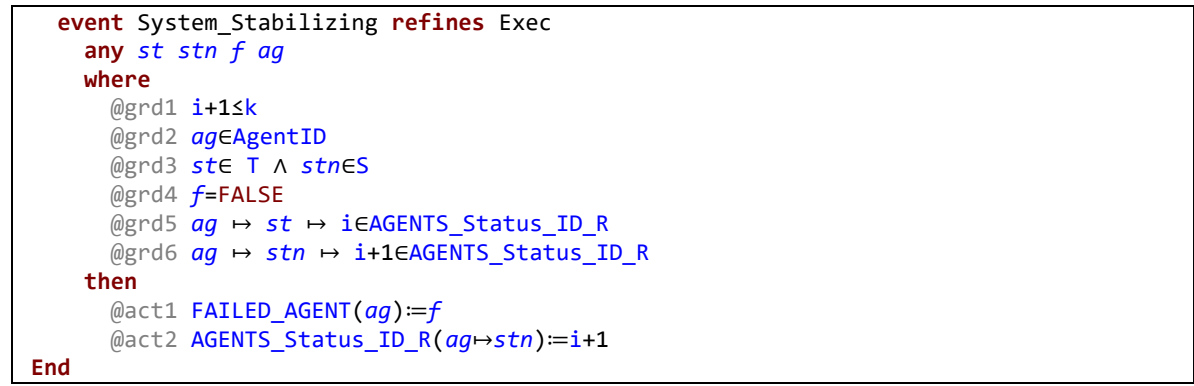

Code 7. A specification of System_Stabilizing event

\subsection{Proof statistics}

In table 1, we can see proof statistics for our model using the Rodin3.2 platform. The statistics give us the proof obligations generated and discharged by the Rodin. The finial development of our model results in 26 POs (Proof obligations). Around (97\%) of them have been proved automatically by the Rodin platform and the rest have been proved manually in the Rodin interactive proving environment.

\begin{tabular}{|c|c|c|c|}
\hline \multirow{2}{*}{ model } & Total & Auto & Manual \\
\cline { 2 - 4 } & $26(100 \%)$ & $25(97 \%)$ & $1(3 \%)$ \\
\hline Abstract & 10 & 10 & 0 \\
\hline Refinement & 16 & 15 & 1 \\
\hline
\end{tabular}

Table 1. Proof Obligations of our model.

\section{Conclusion}

In this paper; we introduced a model in distributed cyber physical systems, definition of self-stabilizing approach for fault tolerance and Event-B as formal method for system-level modelling and analysis. The subject of self-stabilization is so important, and interesting as well, that within the past few years there has been many papers, as well as some workshops devoted entirely to this subject.

Self-stabilizing is an elegant approach for designing a class of fault-tolerant which could use it in distributed Cyber physical system. A self-stabilizing is guaranteed to eventually converge to a legitimate state after a transient fault. However, even a minor transient fault can cause vast disruption in the system before legitimacy is reached. 
When we use stabilizing approach, we can build fault-tolerant DCPS, which allow discussing and defining invariant sets of states describing safety properties and stable sets of states under normal system operation describing progress properties.

This paper presents Event-B methodology which allows us to define a kind of modelling methodology by write the correct mathematical notions; wherefore we can apply Event-B in modelling many different complex projects, but we should choose carefully invariants and variables to ease effort of proof. As well as the Rodin tool offers reactive environment for constructing and analyzing models as do most modern integrated development environments, and provides integration between modelling and proving whereas this is important feature for the developers to focus on the modelling task without switch between different tools to check proving in same time.

In the future work, we want to show that this approach (Event-B) can be used in a number of other applications of controlling different types of systems.

\section{Acknowledgments}

This work was supported by the Ministry of Education, Youth and Sports of the Czech Republic within the National Sustainability Programme project No. LO1303 (MSMT-7778/2014) and also by the European Regional Development Fund under the project CEBIA-Tech No. CZ.1.05/2.1.00/03.0089 and also by the Internal Grant Agency of Tomas Bata University under the project No. IGA/FAI/2018/019.

\section{References}

[1] Volkan, G.; Steffen, P.; Givargis, T. \& Vahid, F. (2014). A Survey on Concepts, Applications, and Challenges in Cyber-Physical Systems. Available from: https://www.ics.uci.edu/ givargis/pubs/J24.pdf.

[2] Bhandari, G.P. \& Gupta, R. (2018). Safety-Critical, Dependable, and Fault-Tolerant Cyber-Physical Systems. Available from: https://www.igi-global.com/chapter/safety-critical-dependable-and-fault-tolerant-cyber-physicalsystems/204667.

[3] Morgan, C.C. \& McIver, A.K. (2006). Programming-Logic Analysis of Fault Tolerance: Expected Performance of Self-stabilization. Available from: http://www.cse.unsw.edu.au/ carrollm/probs/Papers/McIver-06a.pdf.

[4] Saifullah, A. (2012). Self-Stabilization in the Distributed Systems of Finite State Machines; Washington University in St. Louis.

[5] Johnson, T.T. (2010). Fault-tolerant distributed cyber-physical systems: two case studies. University of Illinois at Urbana-Champaign.

[6] Ding, Y. (2014). Fault Tolerance in Distributed Systems Using Self-Stabilization, Clemson University.

[7] Tricaud, Ch. \& Chen, Y.Q. (2012). Optimal Mobile Sensing and Actuation Policies in Cyber-physical Systems, Springer Verlag London.

[8] Roxana, R.B. \& Dulf, E.H. (2017). Fault-tolerant Control of a Cyber physical System. IOP Conference Series: Materials Science and Engineering, Volume 261, conference 1. Available from http://iopscience.iop.org/article/10.1088/1757-899X/261/1/012003/pdf.

[9] Koren, I. \& Krishna, C. (2007). Fault tolerant systems. Elsevier.

[10] Atallah, M.J. \& Blanton, M. (2010). Algorithms and Theory of Computation Handbook, Taylor and Francis Group, LLC.

[11] Nikolov, D. (2015). Fault Tolerance for Real-Time Systems. Lund University.

[12] Neggazi, B. (2015). Self-stabilizing algorithms for graph parameters. Universit'e Claude Bernard Lyon. Available from https://tel.archives-ouvertes.fr/tel-01303138

[13] Ali, A.A.; Jasek, R.; Krayem, S.; Chramcov, B. \& Zacek, P. (2018). Improved Adaptive Fault Tolerance Model for Increasing Reliability in Cloud Computing Using Event-B. In: Silhavy R. (eds) Cybernetics and Algorithms in Intelligent Systems. CSOC2018 2018. Advances in Intelligent Systems and Computing, vol 765. Springer, Cham,

[14] Filaretov, V.; Zhirabok, A.; Zuev, A. \& Protsenko, A. (2016). Method of Fault Identification in Mechatronic Systems, Proceedings of the 27th DAAAM International Symposium, pp.0312-0318, B. Katalinic (Ed.), Published by DAAAM International, ISBN 978-3-902734- 08-2, ISSN 1726-9679, Vienna, Austria

[15] Filaretov, V.; Zhirabok, A.; Zuev, A. \& Protsenko, A. (2016). Identification of Faults in Nonlinear Dynamic Systems, Proceedings of the 26th DAAAM International Symposium, pp.0470-0477, B. Katalinic (Ed.), Published by DAAAM International, ISBN 978-3-902734- 07-5, ISSN 1726-9679, Vienna, Austria 